Nevşehir Bilim ve Teknoloji Dergisi Cilt 4(2) 26-34 2015

DOI: 10.17100/nevbiltek.211037

URL: http://dx.doi.org/10.17100/nevbiltek.211037

\title{
Some Relations Among the Largest Eigenvalues of Product Matrix and Graph Matrices
}

\author{
Sezer Sorgun ${ }^{1}$, Hatice Topcu ${ }^{1 *}$, Kahraman Birgin ${ }^{2}$, Hakan Küçük ${ }^{1}$ \\ ${ }^{1}$ Department of Mathematics, Nevşehir Haci Bektaş Veli University, Nevşehir, Turkey \\ ${ }^{2}$ High School of Social Sciences, Aksaray, Turkey
}

Abstract

We define product matrix as $P(G)=A(G) D(G)$, where $A(G)$ is an adjacency matrix and $D(G)$ is a diagonal matrix of vertex degrees of a graph $G$. In this paper, some relations among the spectral radius of product matrix and the largest eigenvalues of graph matrices are obtained. We also give numerical results for them.

Keywords : Graph Matrices, Laplacian, Adjacency, Signless Laplacian, Spectral Radius.

\section{Graf Matrisleri ve Çarpım Matrisinin En Büyük Öz Değerleri}

\section{Arasında Bazı Bağıntılar}

$\ddot{O z}$

$A(G)$ ve $D(G)$ sırasıyla bir $G$ grafının komşuluk matrisi ve nokta derecelerinin bir köşegen matrisi olmak üzere $P(G)=A(G) D(G)$ matrisini tanımlarız. Bu makalede bu çarpım matrisinin spektral yarıçapı ile graf matrislerinin en büyük öz değerleri arasında bazı bağıntılar elde edilmiştir. Ayrıca nümerik sonuçlar da verilmiştir.

Anahtar Kelimeler : Graf Matrisleri, Laplasyan, Komşuluk, İşaretsiz Laplasyan, Spektral yarıçap.

\footnotetext{
"e-mail: hatice.kamit@nevsehir.edu.tr
} 


\section{Introduction}

Let $G=G_{n, m}=(V, E)$ be a simple connected graph with $n$ vertices and $m$ edges. Let $d_{i}$ be the degree of vertex $i$. A degree sequence is a monotonic non-increasing sequence of the vertex degrees of its graph vertices and it is denoted by $\left[d_{1}, d_{2}, \ldots, d_{n}\right]$. A graph which the degrees of all vertices are equal is called a regular graph. If $d_{i}=n-1$, graph is called as complete graph. Let $\Delta=\Delta(G)$ and $\delta=\delta(G)$ be the maximum degree and the minimum degree of the vertices of $G$, respectively. The eigenvalues of $G$ are the eigenvalues of the adjacency matrix $A(G)$ of $G$, denoted as $\lambda_{1}(G) \geq \lambda_{2}(G) \geq \cdots \geq \lambda_{n-1}(G) \geq \lambda_{n}(G)$. The Laplacian matrix of $G$ is $L(G)=D(G)-A(G)$, where $D(G)=\operatorname{diag}\left(d_{1}, d_{2}, \ldots, d_{n}\right)$ is the diagonal matrix of vertex degrees of $G$. It is well known that $L(G)$ is positive semidefinite matrix. That is, all its eigenvalues are nonnegative. Hence, the eigenvalues of $L(G)$ are denoted as $\mu_{1}(G) \geq \mu_{2}(G) \geq \cdots \geq$ $\mu_{n-1}(G) \geq \mu_{n}(G)=0$. Another graph matrix is called the signless Laplacian and shown that $Q(G)=$ $D(G)+A(G)$. The eigenvalues of the signless Laplacian of $G$ are denoted as $q_{1}(G) \geq q_{2}(G) \geq \cdots \geq$ $q_{n-1}(G) \geq q_{n}(G)$. Again, it is also known that $A(G)$ and $Q(G)$ are irreducible nonnegative matrix. Therefore the largest eigenvalues of $A(G)$ and $Q(G)$ are simple eigenvalue according to Perron-Frobenius Theorem. The least eigenvalue of the signless Laplacian of a connected graph is equal to 0 if and only if the graph is bipartite.

There are too many results related to bounds (upper and lower) for the eigenvalues of graph matrices, see $[1-5,7-8,10-12]$. Also, there are some results about the relations between them. Some of these results are given as below.

In [9], it is shown that

$\mu_{1} \leq q_{1}$

the equality holds if and only if $G$ is a bipartite graph. In [13], it is shown that

$2 \lambda_{1} \leq q_{1}$

the equality holds if and only if $G$ is a bipartite graph. One of the conjectures which is given in [15] has been proved in [14] as

$q_{1}-\mu_{1} \leq n-2$

A similar study have been done in [6]. The conjecture stating that

$1-\sqrt{n-1} \leq q_{2}-\lambda_{1}$

has been proved for $n \geq 9$. In [17], it is obtained that

$q_{1}^{\alpha}+\cdots+q_{n}^{\alpha} \geq \mu_{1}^{\alpha}+\cdots+\mu_{n}^{\alpha}$

for any $\alpha, 0 \leq \alpha \leq 1$.

In this context, we define product matrix of a graph $G$ such that $P(G)=A(G) D(G)$. Then, we get $P(G)=\left(p_{i j}\right)$ where 
$p_{i j}=\left\{\begin{array}{lr}d_{j} & \text { if } i \sim j \\ 0 & \text { otherwise }\end{array}\right.$

By induction, one can see that $(I+P(G))^{n-1}>0$. Hence, $P(G)$ is irreducible matrix. Since $P(G)$ is also a nonnegative matrix (but it is not symmetric), the largest eigenvalue (spectral radius) of $P$ is a simple eigenvalue and it is maximal in modulus among all the other eigenvalues. Let's denote the spectral radius of $P(G)$ as $|\beta|$.

In this paper, we present some relations between the $|\beta|$ and the largest eigenvalues of graph matrices (Adjacency, Laplacian, Signless Laplacian). Also, we give some examples to show the numerical results of these relations. For the graph samples, we use Maple11 and we compare our results on more than 1550 graphs.

\section{Main Results}

Theorem 1.[18] If $\mathrm{G}$ is k-regular, then $\mathrm{k}$ is an eigenvalue of $\mathrm{G}$. Its eigenspace is spanned by a vector which all entries are one. Further, $\lambda_{1}(G)$ equals to $\mathrm{k}$.

Theorem 2. [20] Let $\mathrm{d}$ and $\Delta$ be average degree and maximum degree of the graph G, respectively. Then

$\overline{\mathrm{d}} \leq \lambda_{1}(\mathrm{G}) \leq \Delta$

Moreover $\bar{d}=\lambda_{1}(G)$ if and only if $G$ is regular graph. For connected graph $G, \Delta=\lambda_{1}(G)$ if and only if $G$ is a regular graph.

Theorem 3. Let $\mathrm{G}$ be a simple, connected graph. Then,

$\lambda_{1} \leq \sqrt{|\beta|}$

where $\lambda_{1},|\beta|$ are the spectral radius of $A(G)$ and $P(G)$; respectively. Moreover the equality holds in (8) if $\mathrm{G}$ is a regular graph.

Proof. First, assume that $G$ be a non-regular graph. Maximum absolute column sum norm of $A(G)=$ $\left(a_{i j}\right)$ is

$$
\begin{aligned}
\|A\|_{1} & =\max _{1 \leq J \leq n}\left\{\sum_{i}\left\{\left|a_{i j}\right|: \quad v_{i} v_{j} \in E\right\}\right\} \\
& =\max _{1 \leq J \leq n}\left\{d_{j}\right\}
\end{aligned}
$$

and maximum absolute column sum norm of $P(G)=\left(p_{i j}\right)$ is

$$
\begin{aligned}
\|P\|_{1} & =\max _{1 \leq J \leq n}\left\{\sum_{i}\left\{\left|p_{i j}\right|: \quad v_{i} v_{j} \in E\right\}\right\} \\
& =\max _{1 \leq J \leq n}\left\{\sum_{i}\left\{d_{j}: \quad v_{i} v_{j} \in E\right\}\right\} \\
& =\max _{1 \leq J \leq n}\left\{d_{j}^{2}\right\} \\
& =\left\{\max _{1 \leq J \leq n}\left\{d_{j}\right\}\right\}^{2}
\end{aligned}
$$

Let $\Delta=\max _{1 \leq J \leq n}\left\{d_{j}\right\}$. On the other hand we have 
$\lambda_{1} \leq\|A\|_{1}=\Delta$

and

$|\beta| \leq\|P\|_{1}=\Delta^{2}$

Then $\lambda_{1}^{2}$ is also lower bound for $\Delta^{2}$ from (11), But $|\beta|=\inf \left\{\Delta^{2}\right\}$. Hence, we get

$\lambda_{1}^{2} \leq|\beta|$

i.e.

$\lambda_{1} \leq \sqrt{|\beta|}$

For equality in (8) let $G$ be a $k$-regular graph. Then we have $P(G)=k A(G)$ from the definition of matrices. It is easy to see that $\lambda_{1}=\sqrt{|\beta|}$ from Theorem 1 .

Conversely, assume that equality in (8) holds. Then all inequalities must be equalities. From equalities in (11) and (12) we get

$\lambda_{1} \leq \sqrt{|\beta|}=\Delta$

Hence $G$ must be regular graph from Theorem 2

Theorem 4. [9] Let $\mathrm{G}$ be a graph with at least one edge and the maximum degree $\Delta$. Then

$\mu_{1} \geq \Delta+1$

With equality if and only if there exists a vertex being adjacent to all other vertices in G.

Theorem 5. Let $G$ be a graph on n vertices. Then

$\mu_{1} \geq \Delta+\sqrt{|\beta|}$

with equality if and only if $G$ is a complete graph.

Proof. By inequality (12), we have

$|\beta| \leq \Delta^{2}$

i.e.

$\sqrt{|\beta|}+1 \leq \Delta+1$

$\leq \mu_{1} \quad$ (By (13))

Assume that the equality in (14) holds. Then all the inequalities in the above must be equalities. From the equality in (16) and Theorem 4, $G$ must contain at least one vertex which is adjacent all other vertices. On the other hand, we get that $G$ must be a regular graph from the equality in (15) and Theorem 2. Hence, from both arguments we get that $G$ is a complete graph. 
Conversely, assume that $G$ is a complete graph. Since each vertex is adjacent to other vertices, the condition in Theorem 4 holds. Hence we get

$\mu_{1}=\Delta+1=\sqrt{|\beta|}+1$

Lemma 1. [19] Let $A \in M_{n}$ and $\varepsilon>0$ be given. There is a matrix norm $\|$.$\| such that \rho(A) \leq\|A\| \leq$ $\rho(\mathrm{A})+\varepsilon$, where $\rho(\mathrm{A})$ is the spectral radius of matrix $\mathrm{A}$.

Theorem 6. Let $G$ be a simple, connected graph and $\lambda_{1},|\beta|$ be the largest eigenvalues of $A(G)$ and $P(G)$, respectively. Then

$\lambda_{1} \geq \sqrt{\frac{|\beta|}{\|\mathrm{D}(\mathrm{G})\|}}$

Proof. From Lemma 1, we see easily that

$\|A\| \leq \lambda_{1}^{2}(A)$

Since $P(G)=A(G) D(G)$, we have

$$
\begin{aligned}
\|P(G)\| & =\|A(G) D(G)\| \\
& \leq A(G) D(G)
\end{aligned}
$$

and we get

$|\beta| \leq\|P(G)\| \leq \lambda_{1}^{2} \cdot\|D(G)\|$

i.e.

$\lambda_{1} \geq \sqrt{\frac{|\beta|}{\|D(G)\|}}$

from (18).

Remark 1. In Theorem 6, the matrix norm is any norm. The inequality in (17), if we take maximum absolute column or row sum norm, we have

$\lambda_{1} \geq \sqrt{\frac{|\beta|}{\Delta}}$

such that $\Delta=\mathrm{d}_{1} \geq \mathrm{d}_{2} \cdots \geq \mathrm{d}_{\mathrm{n}}=\delta$. If we apply Euclide norm in (17), we have

$\lambda_{1} \geq \frac{\sqrt{|\beta|}}{\sqrt[4]{\sum_{\dot{\mathrm{I}}}^{\mathrm{n}} \mathrm{d}_{\mathrm{i}}^{2}}}$ 


\section{APPENDIX}

Some examples of graphs are examined and their eigenvalues with two decimal are given in the following list.

Tablo 1.

\begin{tabular}{|c|c|c|c|c|c|c|}
\hline$G_{n, m}$ & Degree Sequence & $\lambda_{1}$ & $\beta_{1}$ & $\mu_{1}$ & $q_{1}$ & $\sqrt{\beta_{1}}$ \\
\hline$G_{3,2}$ & {$[1,1,2]$} & 1.41 & 2.00 & 3.00 & 3.00 & 1.41 \\
\hline$G_{3,3}$ & {$[2,2,2]$} & 2.00 & 4.00 & 4.00 & 4.00 & 2.00 \\
\hline$G_{4,3}$ & {$[1,1,1,3]$} & 1.73 & 3.00 & 4.00 & 4.00 & 1.73 \\
\hline$G_{4,4}$ & {$[2,3,1,2]$} & 2.17 & 4.84 & 4.00 & 4.56 & 2.20 \\
\hline$G_{4,5}$ & {$[3,2,2,3]$} & 2.56 & 6.62 & 4.00 & 5.23 & 2.57 \\
\hline$G_{4,6}$ & {$[3,3,3,3]$} & 3.00 & 9.00 & 4.00 & 6.00 & 3.00 \\
\hline$G_{5,4}$ & {$[1,3,2,1,1]$} & 1.84 & 3.61 & 4.17 & 4.17 & 1.90 \\
\hline$G_{5,5}$ & {$[1,1,3,3,2]$} & 2.30 & 5.65 & 4.30 & 4.93 & 2.37 \\
\hline$G_{5,6}$ & {$[4,2,2,2,2]$} & 2.56 & 6.74 & 5.00 & 5.56 & 2.59 \\
\hline$G_{5,7}$ & {$[3,3,3,3,2]$} & 2.85 & 8.27 & 5.00 & 5.77 & 2.87 \\
\hline$G_{5,8}$ & {$[3,4,3,2,4]$} & 3.32 & 11.24 & 5.00 & 6.82 & 3.35 \\
\hline$G_{5,9}$ & {$[4,3,3,4,4]$} & 3.64 & 13.38 & 5.00 & 7.37 & 3.65 \\
\hline$G_{5,10}$ & {$[4,4,4,4,4]$} & 4.00 & 16.00 & 5.00 & 8.00 & 4.00 \\
\hline$G_{6,5}$ & {$[2,2,2,2,1,1]$} & 1.80 & 3.41 & 3.73 & 3.73 & 1.84 \\
\hline$G_{6,6}$ & {$[3,1,2,3,1,2]$} & 2.33 & 5.85 & 4.39 & 4.98 & 2.41 \\
\hline$G_{6,7}$ & {$[2,2,2,2,2,4]$} & 2.50 & 6.53 & 5.23 & 5.48 & 2.55 \\
\hline$G_{6,8}$ & {$[1,2,2,2,4,5]$} & 3.10 & 10.08 & 6.00 & 6.90 & 3.17 \\
\hline$G_{6,13}$ & {$[4,4,5,5,4,4]$} & 4.37 & 19.23 & 6.00 & 8.82 & 4.38 \\
\hline$G_{6,14}$ & {$[5,5,5,4,4,5]$} & 4.70 & 22.20 & 6.00 & 9.46 & 4.71 \\
\hline$G_{6,15}$ & {$[5,5,5,5,5,5]$} & 5.00 & 25.00 & 6.00 & 10.00 & 5.00 \\
\hline$G_{7,6}$ & {$[1,3,2,1,1,3,1]$} & 2.05 & 4.68 & 4.62 & 4.62 & 2.16 \\
\hline$G_{7,7}$ & {$[1,2,2,3,2,2,2]$} & 2.10 & 4.53 & 4.41 & 4.41 & 2.12 \\
\hline$G_{7,8}$ & {$[2,2,3,2,2,3,2]$} & 2.37 & 5.87 & 4.87 & 4.95 & 2.42 \\
\hline$G_{7,9}$ & {$[1,3,2,4,2,4,2]$} & 2.87 & 8.72 & 5.85 & 6.20 & 2.95 \\
\hline$G_{7,10}$ & {$[2,2,5,4,1,4,2]$} & 3.31 & 11.74 & 6.15 & 7.24 & 3.42 \\
\hline$G_{7,19}$ & {$[5,6,6,6,6,5,4]$} & 5.50 & 30.54 & 7.00 & 11.12 & 5.52 \\
\hline$G_{7,20}$ & {$[6,6,6,6,5,5,6]$} & 5.74 & 33.07 & 7.00 & 11.53 & 5.75 \\
\hline$G_{7,21}$ & {$[6,6,6,6,6,6,6]$} & 6.00 & 36.00 & 7.00 & 12.00 & 6.00 \\
\hline & {$[2,1,1,3,1,1,3,2]$} & 2.09 & 4.93 & 4.68 & 4.68 & 2.22 \\
\hline
\end{tabular}

\begin{tabular}{|c|c|c|c|c|c|c|}
\hline$G_{n, m}$ & Degree Sequence & $\lambda_{1}$ & $\beta_{1}$ & $\mu_{1}$ & $q_{1}$ & $\sqrt{\beta_{1}}$ \\
\hline$G_{8,8}$ & {$[2,1,1,2,1,3,4,2]$} & 2.40 & 6.37 & 5.46 & 5.46 & 2.52 \\
\hline$G_{8,9}$ & {$[4,3,1,3,1,3,1,2]$} & 2.66 & 7.63 & 5.60 & 5.76 & 2.76 \\
\hline$G_{8,10}$ & {$[2,3,1,3,3,2,2,4]$} & 2.76 & 8.03 & 5.47 & 5.88 & 2.83 \\
\hline$G_{8,26}$ & {$[7,6,7,6,6,6,6,6]$} & 6.56 & 43.30 & 8.00 & 13.21 & 6.58 \\
\hline$G_{8,27}$ & {$[7,7,6,7,7,6,7,7]$} & 6.77 & 45.96 & 8.00 & 13.58 & 6.78 \\
\hline$G_{8,28}$ & {$[7,7,7,7,7,7,7,7]$} & 7.00 & 49.00 & 8.00 & 14.00 & 7.00 \\
\hline$G_{9,8}$ & {$[1,1,3,1,1,1,3,1,4]$} & 2.28 & 6.05 & 5.44 & 5.44 & 2.45 \\
\hline
\end{tabular}


Sorgun S., Topcu H., Birgin K., Küçük H.

$\begin{array}{lllllll}G_{9,9} & {[1,2,2,4,1,3,3,1,1]} & 2.39 & 6.23 & 5.39 & 5.39 & 2.49 \\ G_{9,10} & {[1,3,2,1,3,2,2,4,2]} & 2.60 & 7.64 & 5.26 & 5.79 & 2.76 \\ G_{9,11} & {[1,2,4,2,4,2,2,3,2]} & 2.80 & 8.24 & 5.67 & 6.01 & 2.87 \\ G_{9,25} & {[5,4,6,5,4,7,6,4,5]} & 5.69 & 33.09 & 8.65 & 11.66 & 5.75 \\ G_{9,26} & {[5,5,6,5,5,8,6,6,6]} & 5.89 & 35.36 & 9.00 & 12.09 & 5.99 \\ G_{9,27} & {[5,7,6,6,6,7,6,7,4]} & 6.12 & 38.11 & 8.77 & 12.44 & 6.17 \\ G_{9,34} & {[8,8,6,7,8,8,8,8,7]} & 7.60 & 58.10 & 9.00 & 15.29 & 7.62 \\ G_{9,35} & {[8,8,8,8,8,7,8,7,8]} & 7.79 & 60.87 & 9.00 & 15.62 & 7.80 \\ G_{9,36} & {[8,8,8,8,8,8,8,8,8]} & 8.00 & 64.00 & 9.00 & 16.00 & 8.00 \\ G_{10,9} & {[2,3,1,3,3,1,2,1,1,1]} & 2.10 & 4.82 & 4.17 & 4.17 & 2.19 \\ G_{10,10} & {[2,3,1,1,2,3,1,3,1,3]} & 2.35 & 6.10 & 4.17 & 5.08 & 2.46 \\ G_{10,11} & {[2,2,4,2,2,1,2,2,2,3]} & 2.51 & 6.92 & 5.20 & 5.60 & 2.63 \\ G_{10,20} & {[3,4,4,4,4,3,3,6,4,5]} & 4.21 & 18.56 & 7.62 & 8.85 & 4.30 \\ G_{10,31} & {[5,7,9,6,6,6,6,4,6,7]} & 6.39 & 41.87 & 9.05 & 13.23 & 6.47 \\ G_{10,32} & {[6,8,7,6,7,6,6,6,8,4]} & 6.58 & 44.21 & 9.60 & 13.44 & 6.64 \\ G_{10,33} & {[5,6,7,7,8,7,5,7,8,6]} & 6.74 & 46.30 & 9.55 & 13.73 & 6.80 \\ G_{10,34} & {[7,7,4,7,9,8,5,7,7,7]} & 7.01 & 50.22 & 9.62 & 14.34 & 7.08 \\ G_{10,41} & {[9,7,8,9,9,8,8,7,9,8]} & 8,25 & 68.52 & 10.00 & 16.61 & 8.27 \\ G_{10,42} & {[8,8,9,8,9,9,8,9,8,8]} & 8.42 & 71.14 & 10.00 & 16.89 & 8.43 \\ G_{10,43} & {[9,8,9,9,9,9,8,8,9,8]} & 8.62 & 74.52 & 10.00 & 17.29 & 8.63 \\ G_{10,44} & {[9,9,9,9,9,8,9,9,8,9]} & 8.81 & 77.80 & 10.00 & 17.65 & 8.82 \\ G_{10,45} & {[9,9,9,9,9,9,9,9,9,9]} & 9.00 & 81.00 & 10.00 & 18.00 & 9.00\end{array}$

\section{MAPLE CODE}

We describe below some Maple -Code which we use to verify some statements on some sample graphs. We use the mathematical software Maple 11 by Maplesoft. The (standard) commands for graphs are in a package called "networks" and the commands for linear algebra are in a package called "linalg". Hence, one should include these two packages :

with(linalg); with(networks);

The command Adjacency Matrix $(\mathrm{G})$ compute the adjacency matrix of a graph G. There is no command for the Laplacian or the signless Laplacian in Maple. So, we have defined the Laplacian matrix and the signless Laplacian matrix by the following procedure :

$\operatorname{Deg} G:=\operatorname{proc}(G)$ local $i, d, n, m$;

$m:=\operatorname{AdjacencyMatrix}(G)$;

$n:=$ LinearAlgebra $[$ RowDimension $](\operatorname{convert}(m$, Matrix $))$ :

$d:=\operatorname{Matrix}(n, n, 0)$;

For $i$ from 1 to $n$ do

$d[i, i]:=\operatorname{add}(m[i, j], j=1 . . n) ;$

od:

returnd;

end proc: 
$L a p G:=\operatorname{proc}(G)$

return DegG( $G)$ - AdjacencyMatrix $(G)$;

end proc:

SignlessLapG $:=\operatorname{proc}(G)$

return $\operatorname{Deg} G(G)+$ AdjacencyMatrix $(G)$;

end proc:

We have also defined product matrix of a graph $\mathrm{G}$ as below :

$\operatorname{Product} G:=\operatorname{proc}(G)$

return multiply(AdjacencyMatrix $(G), \operatorname{Deg} G(G))$;

end proc:

We have used two packages "with(RandomGraphs)" and "with(GraphTheory)" to examine random graphs.

$G:=$ RandomGraph $(n, m$, connected $)$;

evalf (eigenvals (AdjacencyMatrix ( $G))$ );

evalf (eigenvals (ProductG $(G))$ );

evalf (eigenvals $(\operatorname{Lap} G(G)))$;

evalf (eigenvals(SignlessLapG $(G))$ );

\section{Acknowledgement}

The authors are thankful to the Office of Nevşehir Hacı Bektaş Veli University Scientific Research Project (BAP)

\section{References}

[1] Brualdi R.A., Hoffman A.J., "On the spectral radius of (0,1) matrix”, Linear Algebra Appl., 65, 133-146, 1985.

[2] Stanley R.P., "A bound on the spectral radius of graphs with e edges", Linear Algebra Appl., 67, 267-269, 1987.

[3] Hong Y., "A bound on the spectral radius of graphs", Linear Algebra Appl., 108, 133-140, 1988.

[4] Das K.C., "A characterization of graphs which archive the upper bound for the largest Laplacian eigenvalue of graphs", Linear Algebra Appl., 376, 173-186, 2004.

[5] Das K.C., Kumar P. "Bounds on the greatest eigenavlue of graphs", Indian J. Pure Appl. Math., 34(6), 917-925, 2003. 
[6] Das K.C., "Proof of conjecture involving the second largest signless Laplacian eigenvalue and the index of graphs", Linear Algebra Appl., 435, 2420-2424, 2011.

[7] Zhang X.-D., "Two sharp upper bounds for the Laplacian eigenvalues", Linear Algebra Appl., 376, 207-213, 2004.

[8] Berman A., Zhang X.-D. "On the spectral radius of graphs with cut vertices”, J. Combin. Thoery Series B, 83, 223-240, 2003.

[9] Zhang X.-D., Luo R. "T he spectral radius of triangle-free graphs", Australas J. Combin., 26, 33-39, 2002.

[10] Guo J.-M., "A new upperbound for the Laplacian spectral radius of graphs", Linear Algebra Appl., 400, 61-66, 2005.

[11] Chen Y.,Wang L. "Sharp bounds for the largest eigenvalue of the signless Laplacian of a graph", Linear Algebra Appl., 433, 908-913, 2010.

[12] Lu M., Liu H., Tian F. "An improved upper bound for the Laplacian spectral radius of graphs", Discrete Math., 309, 6318-6321, 2009.

[13] Chen Y., "Properties of spectra of graphs and line graphs", Appl. Math. J. Ser. B, 3, 371-376, 2002.

[14] Feng L., Yu G. "On three conjectures involving of the signless Laplacian spectral radius of graphs”, Pub. De L'Institute Mathematique, 85(99), 35-38, 2009.

[15] Cvetkovic D., Rowlinson P., Simic S.K., "Eigenvalue bounds for the signless Laplacian ", Publ. Inst. Math. (Beograd), 81(95), 11-27, 2007.

[16] Aouchiche M., Hansen P., “A survey of automated conjectures in spectral graph theory”, Linear Algebra Appl., 432, 2293-2322, 2010.

[17] Akbarietal S., "A relation between the Laplacian and signless Laplacian eigenvalues of a graph", J. Algebra Combin., 32, 459-464, 2010.

[18] Godsil C., Royle G., “Algebraic Graph Theory”, Springer Graduate Texts in Mathematics, Vol. 207, 2002.

[19] Horn R.A.., Johnson R., "Matrix Analysis”, Cambridge University Press, New York, 1985.

[20] Cvetkovic D., Rowlinson P., Simic S.K., "An Introduction to the Theory of Graph Spectra", Cambridge University Press, New York, 2010. 